\title{
Le végétalisme éthique : une « particularité protégée » dans le droit britannique sur les discriminations
}

Jo Carby-Hall

\section{OpenEdition}

Journals

Édition électronique

URL : https://journals.openedition.org/rdctss/1269

DOI : $10.4000 /$ rdctss. 1269

ISSN : 2262-9815

Éditeur

Centre de droit comparé du travail et de la sécurité sociale

Édition imprimée

Date de publication : 1 avril 2020

Pagination : 212-217

ISSN : $2117-4350$

\section{Référence électronique}

Jo Carby-Hall, «Le végétalisme éthique : une « particularité protégée » dans le droit britannique sur les discriminations », Revue de droit comparé du travail et de la sécurité sociale [En ligne], 1 | 2020, mis en ligne le 01 novembre 2021, consulté le 11 novembre 2021. URL : http://journals.openedition.org/ rdctss/1269; DOI : https://doi.org/10.4000/rdctss.1269

\section{(c) (i) $\odot$}

Revue de droit comparé du travail et de la sécurité sociale est mise à disposition selon les termes de la Licence Creative Commons Attribution - Pas d'Utilisation Commerciale - Pas de Modification 4.0 International. 


\section{JO CARBY-HALL}

UNIVERSITÉ DE HULL

\section{LE VÉGÉTALISME ÉTHIQUE : UNE « PARTICULARITÉ PROTÉGÉE » DANS LE DROIT BRITANNIQUE SUR LES DISCRIMINATIONS}

Après une audience préliminaire les 2 et 3 janvier 2020, I'Employment Tribunal, devant le juge Robin Postle, a statué ${ }^{1}$ que le végétalisme éthique est une croyance philosophique et, constitue dès lors une " particularité protégée " au titre de la section 10 de l'Equality Act de 2010. Cette décision va avoir des implications pour les entreprises et autres organisations. Avant d'examiner ci-dessous ces implications, il convient d'abord de définir le terme de «végétalisme».

D'après la Vegan Society, le végétalisme est une philosophie et un mode de vie qui consistent à exclure, dans la mesure du possible, toute forme d'exploitation des animaux et de cruauté envers eux, que ce soit pour se nourrir, se vêtir ou à d'autres fins, et également à promouvoir le développement et l'utilisation d'alternatives en faveur du bien-être des animaux, des humains et de l'environnement. En termes de régime alimentaire, il s'agit d'éviter tous les produits entièrement ou partiellement dérivés de l'animal.

Le point commun de tous les végétaliens réside ainsi, d'une part dans une alimentation basée sur le végétal, excluant la viande, les fruits de mer, les œufs, le poisson, les insectes, les produits laitiers, le miel, ainsi que tous les produits dérivés, et d'autre part dans la proscription des produits testés sur les animaux, et des sites où se déroulent des spectacles animaliers².

\section{I - LES FAITS DE L'AFFAIRE JORDI CASAMITJANA V. THE LEAGUE AGAINST CRUEL SPORTS}

Le requérant, M. Jordi Casamitjana, est un végétalien éthique. Ceci signifie qu'il n'a pas seulement adopté un régime alimentaire végétalien mais qu'il s'oppose aussi à l'utilisation et à l'exploitation abusive des animaux quel qu'en soit le but. Le végétalisme éthique affecte donc tous les aspects de sa vie et pas seulement son alimentation. Les défendeurs, la League Against Cruel Sports, est une association de protection des animaux qui fait campagne contre les sports sanguinaires, comme par exemple la chasse aux lièvres, lapins, renards, cerfs, oiseaux, etc. Le requérant

1 A la suite d'une audience préliminaire les 2 et 3 janvier 2020 dans l'affaire Jordi Casamitjana $\checkmark$ The League Against Cruel Sports.

2 Source: The Vegan Society. En Grande-Bretagne, tous les médicaments sont testés sur des animaux avant d'être déclarés propres à la consommation par les humains. Puisqu'un végétalien mort n'est utile à personne la Vegan Society ne recommande pas de refuser les médicaments prescrits par un médecin! 
avait été employé à deux reprises par les défendeurs de 2004 à 2007, puis du 29 août 2016 au 6 avril 2018.

Alors qu'il était salarié de la League, le requérant formula une demande de renseignements concernant le régime des retraites sélectionné par l'association. Cette démarche révéla que lesdits fonds de retraite n'étaient pas éthiques dans la mesure où ils étaient utilisés par des compagnies qui nuisaient aux animaux.

Cette situation était en totale contradiction avec la philosophie du requérant qui, pour sa part, agissait de manière à s'assurer que ses contributions de retraite étaient bel et bien investies dans des fonds éthiques. II estima donc que les résultats de son enquête constituaient un affront aux convictions de ses collègues de travail qui ignoraient la destination de leurs contributions. M. Jordi Casamitjana les en informa par courriel et contacta en particulier le personnel des défendeurs, auquel il fit part de sa demande de transfert de ses contributions, leur offrant dix alternatives de fonds et leur exprimant son opinion.

Les défendeurs le licencièrent sans préavis pour faute grave, arguant du fait qu'il avait transgressé les instructions de la direction qui lui avait pourtant intimé, expressément et à plusieurs reprises, de ne pas envoyer de courriels ni de donner de conseils d'ordre financier.

Se basant sur un paragraphe de la lettre de licenciement envoyée par les défendeurs - qui stipulait : «D'après votre interprétation et votre philosophie, vous agiriez encore de la même manière si l'occasion se présentait. Je considère que votre courriel tendancieux, en raison de votre éthique, pourrait inciter vos collègues à modifier leurs dispositions en matière de retraite »- le requérant allégua qu'il avait été victime de discrimination directe ou indirecte, et qu'il était harcelé en raison de sa philosophie éthique végétalienne.

Cette affaire appela à se prononcer sur deux points :

- Tout d'abord le concept de philosophie éthique végétalienne, puis le licenciement. Le présent commentaire ne concernera que le premier point déjà jugé à l'audience préliminaire, à savoir la philosophie éthique végétalienne. Pour avancer l'affaire, l'Employment Judge Riobin Postle devait décider si le « végétalisme éthique » du requérant était une « particularité protégée » au titre de l'Equality Act de 2010.

- Concernant le second aspect, à savoir le licenciement, l'audience se tiendra à une date ultérieure. L'Employment Tribunal devra alors déterminer si, en licenciant le requérant pour faute grave, la League l'a traité défavorablement en raison de sa philosophie éthique du végétalisme.

\section{II - LES DISPOSITIONS DU DROIT BRITANNIQUE EN MATIÈRE DE DISCRIMINATION, VICTIMISATION ET HARCÈLEMENT}

La section 10 de l'Equality Act de 2010 prévoit la protection contre les discriminations basées sur des «particularités protégées ( (protected characteristics). Celles-ci comprennent le sexe, la race, l'âge, l'orientation sexuelle, le changement de sexe, le mariage et le partenariat civil, la grossesse et la maternité, ainsi que la discrimination en matière de religion, de croyance ou même de non croyance. 
Le terme de « croyance » est interprété dans le sens le plus large et recouvre tant la croyance religieuse que la « croyance philosophique ", "la référence à une croyance comprenant la référence à la non croyance $»^{3}$.

La législation est rédigée de manière flexible dans la mesure où la discrimination, dans le cas d'une "particularité protégée », est automatiquement illégale. En accord

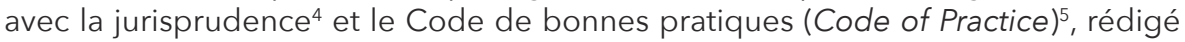
en vertu des dispositions de l'Equality Act de 2010, cinq conditions doivent être remplies pour que le terme de "croyance philosophique " soit admissible au titre $d^{\prime} u n e$ " particularité protégée $»^{6}$ :

1 - Il faut que la « croyance » soit authentique.

2 - Il faut que ce soit une croyance philosophique, et non une opinion ou un point de vue basé sur des informations diffusées dans les médias ou autres.

3 - La croyance doit avoir un certain " poids » et représenter un aspect crucial de l'existence humaine et de son comportement.

4 - La croyance doit comporter un certain degré de sérieux, d'importance, de cohésion et de force.

5 - Enfin, cette croyance doit être respectée dans une société démocratique, ne pas être incompatible avec la dignité humaine et ne pas enfreindre les droits fondamentaux d'autrui.

\section{III - LA DÉCISION DE L’EMPLOYMENT TRIBUNAL}

A l'audience, les défendeurs étaient prêts à concéder les cinq conditions susmentionnées. L'Employment Judge préféra parvenir à ses propres conclusions au terme d'un raisonnement selon lequel le végétalisme éthique remplissait effectivement les cinq conditions.

II déclara donc que le végétalisme éthique était bien une " particularité protégée » en vertu de l'Equality Act de 2010, en particulier parce qu'il mérite le respect dans une société démocratique et n'est pas incompatible avec la dignité humaine.

S'agissant du végétalisme éthique reconnu comme faisant partie des " particularités protégées ", l'affaire Jordi Casamitjana v. The League Against Cruel Sports est, semble-t-il, la première à être entendue par des Employment Tribunals et des Cours de justice britanniques.

La condition déterminante pour une issue du procès favorable au demandeur était que le végétalisme éthique soit reconnu comme " particularité protégée » en

3 Equality Act, 2010, s.10.

4 Voir Grainger v. Nicholson [2010] IRLR 4, p. 24.

5 https://.equalityhumanrights.com/sites/default/filesemployercode.pdf

6 Notons que ces tests ont été affinés et développés à la suite des affaires Harrow v. Chief Constable of Dorset Police [2016] IRLR 481, et Gray v. Mulberry Co. (Design) [2018] IRLR 893. 
vertu des dispositions de l'Equity Act de 20107', ce qui constitua d'ailleurs I'unique question traitée à l'audience préliminaire.

A l'audience devant l'Employment Tribunal, l'attachement de M. Casamitjana au végétalisme éthique fut largement démontré. Ce dernier préférait se déplacer à pied plutôt que prendre l'autobus - qui pourrait tuer des insectes sur son pare-brise et dont la vitesse pourrait blesser des renards, des chats, des chiens, des oiseaux et bien d'autres animaux susceptibles de traverser la rue.

En outre, M. Casamitjana ne mangeait ni ne portait aucun produit issu du monde animal. Un grand nombre de preuves irréfutables avancées dans cette affaire permettaient donc d'établir que le demandeur était un végétalien éthique engagé et que son végétalisme éthique était une croyance fondamentale. Sa philosophie « imprégnait tous les aspects de sa vie ». Autrement dit, il « la » vivait. Le poids de la preuve est ici très important ${ }^{8}$.

A la sortie de l'audience, M. Casamitjana a déclaré : " Je suis extrêmement heureux de l'issue de ce procès et des conclusions du Juge qui a parfaitement compris ce qu'est le végétalisme éthique. Je ne m'attendais pas à ce qu'une décision intervienne dès aujourd'hui, mais le poids écrasant des preuves que nous avons fournies a suffi pour que le Juge parvienne à la conclusion que je suis bien le végétalien éthique que je professe être et que le végétalisme éthique est une "croyance philosophique non religieuse protégée" $"$ ".

Les travailleurs ont le droit de ne pas être victimes d'intimidation, de harcèlement ou de discrimination dans l'emploi du fait de «particularités protégées ». Dès lors, les employeurs qui pratiquent la discrimination, l'intimidation ou le harcèlement contre les végétaliens éthiques seraient passibles du même traitement par les tribunaux et les Cours de justice que ceux qui profèrent des insultes sexistes ou raciales à caractère discriminatoire. Le végétalisme éthique, en tant que « théorie philosophique ", est inhérent à la vie quotidienne d'un individu au même titre qu'une croyance religieuse $^{10}$.

La dernière remarque au sujet de cette affaire concerne le fait que la décision émanant d'un Employment Tribunal ne peut pas constituer un précédent en Common Law. En effet, aucun tribunal ni Cour de justice ne sera lié par cette décision en vertu de la doctrine du précédent judiciaire.

7 Ni l'affaire, ni la décision, n'ont été publiées à la date de rédaction de cet article (février 2020). Les détails donnés proviennent cependant de sources fiables.

8 Voir Conisbee v. Crossley Farms Ltd., affaire dans laquelle une revendication pour cause de végétalisme éthique ne fut pas retenue comme " particularité protégée ». La philosophie alimentaire d'un végétalien qui la choisit pour raisons de santé diffère clairement de celle d'un végétalien éthique, dont la position morale reconnaît que les animaux sont des êtres doués de sensibilité et qu'il est donc moralement indéfendable de les faire souffrir et de les exploiter.

9 http://www.personnetoday.comhr/tribunal-rules-thst-ethica

10 Ceci est en concordance avec les dispositions de l'Art. 9 de la Convention Européenne des Droits de l'Homme : «liberté de pensée, de conscience et de religion ». 
Toutefois, cette décision peut constituer une évidence probante. N'oublions pas que les défendeurs étaient d'accord pour accepter que le végétalisme éthique soit considéré comme une " particularité protégée » (voir ci-dessus) ; il est donc improbable qu'ils fassent appel contre cette décision à l'Employment Tribunal.

\section{IV - LES RÉPERCUSSIONS DE L'AFFAIRE CASAMITJANA POUR LES ENTREPRISES ET LES ORGANISATIONS}

De nombreuses répercussions découlent de cette décision. La politique des entreprises et autres organisations devra probablement être réexaminée, voire même révisée le cas échéant, pour se conformer aux exigences légales en matière de soutien des travailleurs végétaliens éthiques au sein de leur entreprise.

Ces obligations pourront comprendre l'intégration de plats végétaliens dans les menus des cantines, cafétérias et restaurants. II faudra également ajouter des collations végétaliennes pour les réunions professionnelles.

En outre, il est possible que les patrons soient contraient de vérifier que les équipements de bureau et uniformes sont bien conformes aux exigences végétaliennes.

Par ailleurs, les employeurs vont devoir afficher leur respect envers le végétalisme éthique et veiller à ce qu'il n'y ait pas de discrimination contre les travailleurs pour des raisons de croyances éthiques.

Certains employeurs vont être amenés à revoir le mode de travail pour tenir compte des végétaliens éthiques. Par exemple, un caissier de supermarché végétalien pourra-t-il être justifié de refuser de passer à la caisse un produit à base de viande, de poisson, de volaille, etc. ?

Il est également possible que l'impact de cette décision s'étende au-delà de l'emploi en tant que tel et implique, par exemple, les prestataires de services qui assurent l'approvisionnement des repas végétaliens dans les écoles, hôpitaux, prisons, etc.

Enfin, les entreprises et organismes seront contraints de réviser leur règlement de manière à ce que la victimisation et le harcèlement soient effectivement contrôlés. Les remarques sur le végétalisme de certains pourraient s'avérer offensantes et constituer alors une discrimination fondée sur les croyances philosophiques.

Pour les végétaliens inter se, la décision dans l'affaire Casamitjana aura pour effet de promouvoir le végétalisme. Il est fort probable qu'un nombre accru de végétaliens surgisse et que l'on assiste à une progression du nombre de défenseurs de la protection animale, de la santé et de l'environnement. Les végétaliens se sentiront habilités et libres d'exprimer leurs opinions sans crainte de représailles.

Mais surtout, cette décision en encouragera d'autres à réclamer la protection des tribunaux et Cours de justice en vertu de leur croyance philosophique.

De plus, cette décision pourrait ouvrir les écluses pour les travailleurs qui professent des convictions fermes dans d'autres domaines - féminisme, communisme, pacifisme, etc. - et qui cherchent à faire reconnaître leurs convictions 


\section{ROYAUME-UNI}

comme philosophies. Le végétarisme est proche du végétalisme; pourquoi alors ne pourrait-il pas être reconnu comme une philosophie s'il peut satisfaire aux cinq critères énoncés ci-dessus?

Pour conclure, quelle que soit la décision de l'Employment Tribunal à propos du licenciement dans cette affaire Casamitjana ${ }^{11}$, il en résultera quelque chose de positif pour le végétalisme: les végétaliens éthiques jouiront à l'avenir d'une meilleure protection.

11 Le 2 mars 2020, The League Against Cruel Sports a conclu un accord amiable avec le requérant, considérant que ce dernier n'aurait pas du être licencié à cause de ses agissements, et affirmant par ailleurs son soutien au végétalisme. 